\title{
Development and Validation of a Nomogram for Preoperative Prediction of Central Compartment Lymph Node Metastasis in Patients with Papillary Thyroid Carcinoma and Type 2 Diabetes Mellitus
}

This article was published in the following Dove Press journal:

Cancer Management and Research

\author{
Chao $\mathrm{He}^{1}$ \\ Yiqiao $\mathrm{Lu}^{\mathrm{l}, *}$ \\ Binqi Wang ${ }^{2, *}$ \\ Jie $\mathrm{He}^{3}$ \\ Haiguang Liu' \\ Xiaohua Zhang' \\ 'Department of Surgical Oncology, The \\ First Affiliated Hospital of Wenzhou \\ Medical University, Wenzhou, Zhejiang, \\ People's Republic of China; ${ }^{2}$ The Second \\ Clinical Medicine Faculty, Zhejiang \\ Chinese Medical University, Hangzhou, \\ Zhejiang, People's Republic of China; \\ ${ }^{3}$ Operating Room, The First Affiliated \\ Hospital of Wenzhou Medical University, \\ Wenzhou, Zhejiang, People's Republic of \\ China
}

*These authors contributed equally to this work
Correspondence: Xiaohua Zhang; Haiguang Liu

Department of Surgical Oncology, The First Affiliated Hospital of Wenzhou Medical University, Ouhai, Wenzhou, Zhejiang, 325000, People's Republic of China

Tel +8657755579463

Email m13587689297@I63.com;

liuhgwzmc@yahoo.com
Purpose: To develop and validate a nomogram to predict central compartment lymph node metastasis in PTC patients with Type 2 Diabetes.

Patients and Methods: The total number of enrolled patients was 456. The optimal cut-off values of continuous variables were obtained by ROC curve analysis. Significant risk factors in univariate analysis were further identified to be independent variables in multivariable logistic regression analysis, which were then incorporated and presented in a nomogram. The ROC curve analysis was performed to evaluate the discrimination of the nomogram, calibration curves and Hosmer-Lemeshow test were used to visualize and quantify the consistency. Decision curve analysis (DCA) was performed to evaluate the net clinical benefit patients could get by applying this nomogram.

Results: ROC curve analysis showed the optimal cutoff values of NLR, PLR, and tumor size were $2.9204,154.7003$, and $0.95(\mathrm{~cm})$, respectively. Multivariate logistic regression analysis indicated that age, multifocality, largest tumor size, and neutrophil-to-lymphocyte ratio were independent prognostic factors of CLNM. The C-index of this nomogram in the training data set was 0.728 , and 0.618 in the external validation data set. When we defined the predicted possibility $(>0.5273)$ as high-risk of CLNM, we could get a sensitivity of 0.535 , a specificity of 0.797 , a PPV(\%) of 67.7 , and an NPV(\%) of 68.7. Great consistencies were represented in the calibration curves. DCA showed that applying this nomogram will help patients get more clinical net benefit than having all of the patients or none of the patients treated with central compartment lymph node dissection (CLND).

Conclusion: A high level of preoperative NLR was an independent predictor for CLNM in PTC patients with T2DM. And the verified optimal cutoff value of NLR in this study was 2.9204. Applying this nomogram will help stratify high-risk CLNM patients, consequently enabling these patients to be treated with appropriate measures. What is more, we hope to find more sensitive indicators in the near future to further improve the sensitivity and specificity of our nomogram.

Keywords: papillary thyroid carcinoma, type 2 diabetes mellitus, neutrophil-lymphocyte ratio, nomogram, central compartment lymph node metastasis

\section{Introduction}

As reported in a recent study, diabetes mellitus is nowadays known as a chronic disease complicated with high incidence and mortality. ${ }^{1}$ It has been discovered, in a large amount of investigations, that the levels of inflammatory cytokines in 
patients with diabetes such as tumor necrosis factor (TNF)-a, c-reactive protein (CRP), interleukin (IL)-1 etc. are much higher than that of non-diabetic population. ${ }^{2,3}$ In addition, it has been widely admitted that chronic inflammation plays an important role in the occurrence and development of diabetes mellitus as well as the pathogenesis of its complications. Different types of organism cells can secrete various kinds of inflammatory cytokines, which will be transferred and released into blood circulation and will actually have different effects on different tissue types. ${ }^{4}$ What is more, the NLR and PLR values have been detected to be much higher in diabetic groups than that in control groups, and the reason might be the increased and high insulin resistance. ${ }^{5,6}$

In various kinds of cancers in humans, the systemic inflammatory response and tumor micro-environment have recently been detected to have important roles in the activation of tumor cell proliferation, invasion, as well as metastasis. ${ }^{7,8}$ In addition, all these markers in peripheral blood parameters including white blood cell, lymphocyte, neutrophil, platelet counts, monocyte, as well as neutrophil-lymphocyte ratio (NLR), platelet-lymphocyte ratio (PLR) and lymphocyte-monocyte ratio (LMR), and systemic immune-inflammation index (SII), which are indicators of systemic inflammatory response, can be obtained inexpensively and easily, and can further be used to enhance the evaluation of prognosis in many kinds of cancers in humans. The platelet-to-lymphocyte ratio (PLR) and neutrophil-to-lymphocyte ratio (NLR) have been indicated to be reliable and independent prognostic bio-markers in colorectal, esophageal, lung, ovarian, gastric, hepatocellular, and pancreatic cancer. ${ }^{9-16}$ And a great number of studies have shown that the initiation and development of thyroid carcinoma is closely associated with tumor-related inflammation. ${ }^{17}$

A great number of studies have investigated the high level of NLR or PLR as risk factors for CLNM in patients with PTC. ${ }^{18-20}$ But the levels of NLR and PLR are different in normal people and people with diabetes mellitus, as we have mentioned previously. ${ }^{5,6}$ Accordingly, as there have been no studies evaluating the association of NLR and PLR with CLNM in PTC patients complicated with T2DM, we decided to explore the relationship in this study.

\section{Patients and Methods}

\section{Patients' Enrollment}

Patients who were diagnosed with PTC and T2DM, treated with lobectomy of thyroid gland as well as lymph node dissection surgery at the First Affiliated Hospital of Wenzhou
Medical University from January 2017 to November 2020, and at the Zhejiang Provincial People's Hospital from 2020 to 2018, of which the total number was 356 and 100 respectively, were finally enrolled in this study. The clinicopathological information of patients at the First Affiliated Hospital of Wenzhou Medical University was collected as the testing data set and that of patients at the Zhejiang Provincial People's Hospital was collected as the external validation data set. The integration of the information and comparison between two groups are shown in Table 1.

\section{Exclusion Criteria}

1) Patients whose primary lesions indicated malignancy after surgical treatment lacked relevant pathological information of lymph nodes, or who did not undergo lymph node dissection.

2) Patients with unknown or unrecorded course of diabetes and specific medication regimen in the medical records.

3) Patients who could not be scheduled for surgical treatment after evaluation due to unstable blood glucose condition after admission.

4) Patients with a history of thyroid surgery or secondary lymph node metastasis after surgery.

5) The presence of distant metastasis, such as pulmonary metastasis, was suspected or confirmed by preoperative ultrasound, CT or biopsy.

6) Patients with other malignant tumors.

7) Patients with hyperthyroidism and other related diseases.

8) Patients with baseline infectious or known inflammatory conditions (except for thyroid autoimmune inflammation, such as HT).

\section{Data Collection}

All eligible patients selected were given a well-established preoperative assessment, including cervical computed tomography, thyroid ultrasound, blood biochemical testing, testing of thyroid function etc. Baseline demographic characteristics were obtained including age, sex, BMI, duration of diabetes, and medical history was taken (containing the medicine for diabetes), the largest tumor size, laterality, multifocality and presentation of chronic lymphocytic thyroiditis according to the thyroid ultrasound together with cervical CT. We also collected the surgical records (intraoperative and postoperative pathology including the status of CLNM). Peripheral blood samples were routinely obtained within 1 week prior to surgery. The formula of calculating NLR and PLR: 
Table I Baseline Characteristics of the Training Data Set and the External Validation Data Set

\begin{tabular}{|c|c|c|c|}
\hline \multirow[t]{2}{*}{ Variables } & Training Data Set(\%) & Validation Data Set(\%) & \multirow[t]{2}{*}{ P-value } \\
\hline & $N=356(78)$ & $N=100(22)$ & \\
\hline Age & & & 0.412 \\
\hline$\leq 55(\mathrm{Y})$ & $158(44.4)$ & $49(49.0)$ & \\
\hline$>55(Y)$ & 198(55.6) & $51(51.0)$ & \\
\hline Sex & & & 0.789 \\
\hline Female & $237(66.6)$ & $68(68.0)$ & \\
\hline Male & $119(33.4)$ & $32(32.0)$ & \\
\hline \multicolumn{3}{|l|}{ Medicine for diabetes } & \\
\hline Metformin(-) & $191(53.7)$ & $62(62.0)$ & 0.138 \\
\hline Metformin $(+)$ & $165(46.3)$ & $38(38.0)$ & \\
\hline Laterality & & & 0.871 \\
\hline Unilateral & $293(82.3)$ & $83(83.0)$ & \\
\hline Bilateral & $63(17.7)$ & $17(17.0)$ & \\
\hline Multifocality & & & 0.873 \\
\hline Solitary & $257(72.2)$ & $73(73.0)$ & \\
\hline Multiple & $99(27.8)$ & $27(27.0)$ & \\
\hline Hashimoto's thyroiditis & & & 0.947 \\
\hline Absent & $316(88.8)$ & $89(89.0)$ & \\
\hline Present & $40(11.2)$ & $I I(I I .0)$ & \\
\hline Largest tumor size(cm), median (range) & $0.7(0.5-1.2)$ & $0.75(0.5-1.1)$ & 0.593 \\
\hline Duration of diabetes $(\mathrm{Y})$, median (range) & $5(2-10)$ & $5(2-10)$ & 0.523 \\
\hline BMI(kg/m2), median (range) & $24.77(23.03-26.80)$ & $24.40(22.70-27.33)$ & 0.820 \\
\hline $\mathrm{TC}(\mathrm{mmol} / \mathrm{L})$, median (range) & $4.76(3.99-5.56)$ & $5.19(4.26-5.95)$ & 0.207 \\
\hline $\mathrm{TG}(\mathrm{mmol} / \mathrm{L})$, median (range) & $1.85(\mid .22-2.71)$ & $1.88(1.19-2.99)$ & 0.884 \\
\hline Glucose(mmol/L), median (range) & $7.9(6.3-10.8)$ & $7.95(6.3-11.5)$ & 0.946 \\
\hline Albumin(g/L), median (range) & $43.20(40.70-45.70)$ & $43.20(40.55-45.20)$ & 0.685 \\
\hline AGR, median (range) & $1.40(1.30-1.60)$ & $1.40(1.30-1.60)$ & 0.670 \\
\hline Percentage of lymphocytes, median (range) & $0.316(0.258-0.365)$ & $0.327(0.251-0.374)$ & 0.614 \\
\hline $\mathrm{TSH}(\mathrm{mlU} / \mathrm{L})$, median (range) & $1.36(0.95-1.93)$ & $1.43(0.93-1.92)$ & 0.962 \\
\hline \multicolumn{3}{|l|}{ NLR } & \\
\hline Low level $(\leq 2.9204)$ & $238(83.7)$ & $81(81.0)$ & \\
\hline High level (>2.9204) & $58(16.3)$ & $19(19.0)$ & \\
\hline PLR & & & 0.218 \\
\hline Low level $(\leq 154.70)$ & $283(79.5)$ & $85(85.0)$ & \\
\hline High level $(>154.70)$ & $73(20.5)$ & $15(15.0)$ & \\
\hline NLR+PLR (NPS) & & & 0.901 \\
\hline Score 0 & $259(72.8)$ & $75(75.0)$ & \\
\hline Score 1 & $63(17.7)$ & $16(16.0)$ & \\
\hline Score 2 & $34(9.6)$ & $9(9.0)$ & \\
\hline CLNM & & & 0.795 \\
\hline CLNM(-) & $20 I(56.5)$ & $55(55.0)$ & \\
\hline CLNM(+) & $155(43.5)$ & $45(45.0)$ & \\
\hline
\end{tabular}




$$
\begin{aligned}
N L R & =\frac{\text { Neutrophil count }}{\text { lymphocyte count }} \\
P L R & =\frac{\text { platelet count }}{\text { lymphocyte count }} .
\end{aligned}
$$

\section{Statistical Analysis Method}

Categorical variables were expressed as the number of cases, percentages (\%) and continuous variables were indicated with medians and quartile. We used the Chi-squared test to compare the rates. And we used $t$-test for the comparison of data between the two groups (when data were normally distributed) or Mann-Whitney $U$-test (when data were not normally distributed). Reference consultation as well as receiver operating characteristic (ROC) curve were performed to determine the optimal cutoff values of the variables. Then we stratified the patients by the most objective cut-off value calculated. Multivariate analysis using the Logistic regression model with forward elimination was performed based on the results of the univariate analysis $(\mathrm{p}<0.1)$. And odds ratios (ORs) with $95 \%$ confidence intervals (CIs) as well as p-value were calculated. Variables with a p-value $<0.05$ in the multivariate analysis were then selected for developing the nomogram. The AUC of the ROC curve was calculated to quantify the prediction efficiency of the nomogram in both the training data set and external validation data set. We performed calibration curves to visualize and evaluate the consistencies between the actual observed situation and the predicted values. To go a step further, Hosmer-Lemeshow test was performed to evaluate the $p$ values of the calibration curves ( $p>0.1$ was considered as a sign of good consistency). DCA curve can quantify the net benefit at disparate threshold probabilities so as to determine the clinical value of using the nomogram in daily medical practice. A criterion of two-sided $\mathrm{P}<0.05$ was considered to be statistically significant. $\mathrm{R}$ software (version 4.0.3) and Spss22.0 were used to finish all statistical analyses mentioned previously.

\section{Results}

\section{Clinically Relevant Characteristics and Demographics}

In our study, we enrolled a total number of 456 cases with confirmed PTC and T2DM from two medical centers as mentioned previously. All data we obtained were then divided into two groups according to the data source: the training data set $(\mathrm{n}=356,78 \%)$ and external testing data set $(n=100,22 \%)$. Approximately $44 \%$ of the patients in the training data set were $\leq 55$ years old, and $49 \%$ in the external validation data set. In the training data set, the ratio of males to females was about 1:2, similar to that of the external testing data set. We found the bigger proportion of cases were lymph node negative $(56.5 \%$ of the training data set, and $55 \%$ of the external testing data set). The relevant data are summarized and shown in Table 1.

\section{Choosing the Optimal Cutoff Value}

In order to be objective, ROC analysis was then performed to choose the optimal cutoff point of NLR, as shown in Figure 1A (AUC=0.539). The most objective and best cutoff value of NLR for predicting CLNM was 2.9204 with the sensitivity of 0.206 and the specificity of 0.871 . As shown in Figure 1B (AUC $=0.530)$, the best PLR cutoff point was 154.7003 of which the sensitivity was 0.239 and the specificity was 0.821 . As shown in Figure 1C (AUC $=0.671$ ), the best cutoff point of largest tumor size was $0.95(\mathrm{~cm})$ with the sensitivity of 0.535 and specificity of 0.806 .

\section{NPS Calculation}

The NPS was calculated under the following rules: patients showing either elevated NLR $(>2.92)$ or PLR $(>154.7)$ or none were assigned a score of 1 or 0 , respectively. And patients with an elevated NLR (>2.92) and PLR (>154.7) at the same time were assigned a score of 2.

\section{Correlation Between Clinical Characteristics and NLR, PLR in All Cohorts}

On the one hand, as for NLR, the optimal cut-off value was 2.9204. Thus we divided patients into two groups by the cut-off value obtained: group A, low NLR level ( $\leq 2.92)$, including 379 patients $(83.1 \%)$; group B, high NLR level (>2.92), including 77 patients (16.9\%); Table 2 indicates that the level of NLR was obviously associated with percentage of lymphocytes $(\mathrm{P}=0.000)$, PLR $(\mathrm{P}=0.000)$. The lower NLR level was correlated with higher percentage of lymphocytes and a lower level of PLR.

On the other hand, as for PLR, the most objective cutoff point was 154.7003 . Thus we divided patients into two groups by the cut-off value: group A, low PLR level 

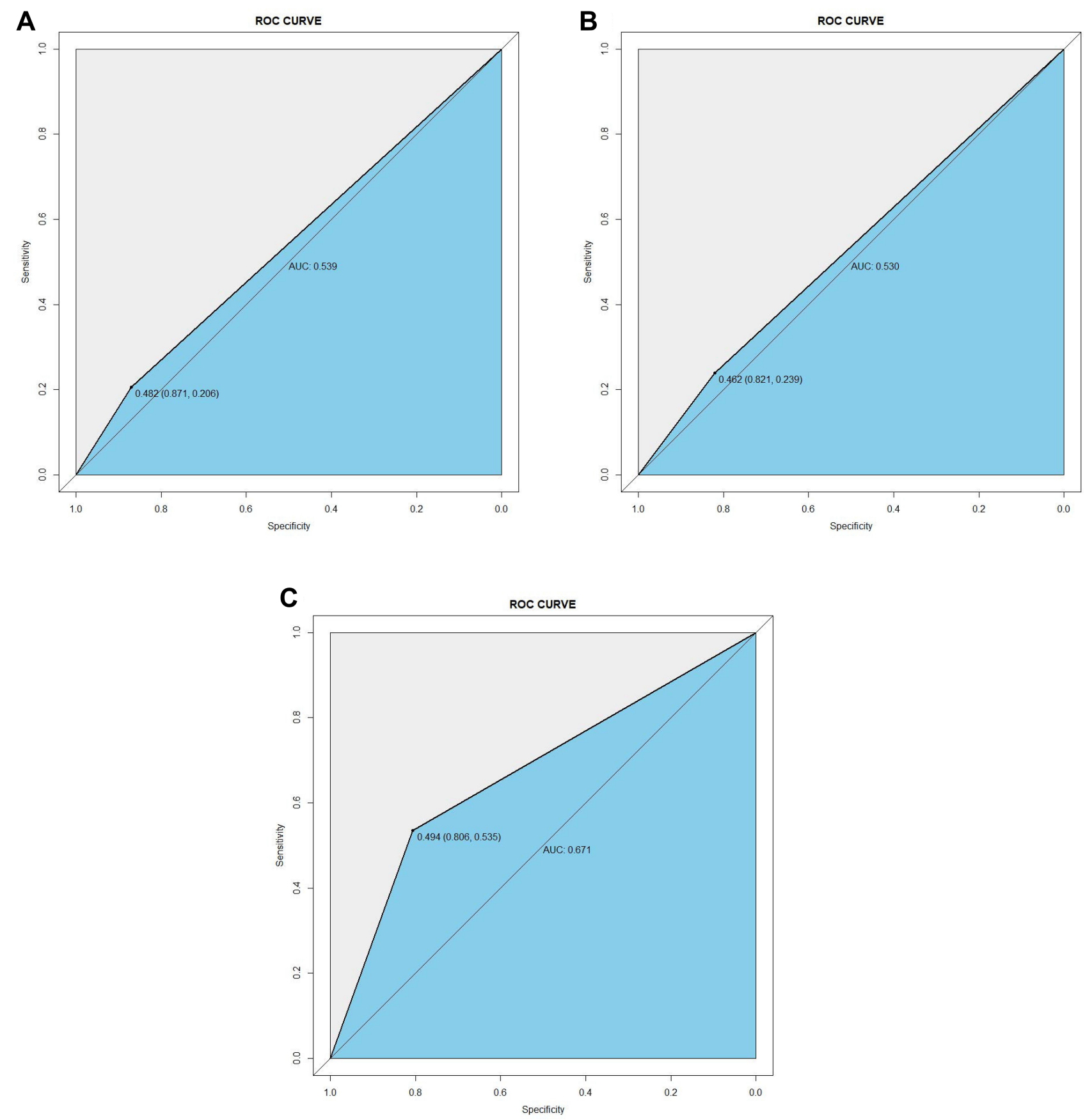

Figure I Receiver operating characteristic (ROC) analysis based on neutrophil-to-lymphocyte ratio (A), platelet-to-lymphocyte (B), and largest tumor size (C).

$(\leq 154.70)$, including 368 patients $(80.7 \%)$; group $\mathrm{B}$, high PLR level (>154.70), including 88 patients (19.3\%); Table 3 shows that the level of PLR was significantly correlated with sex $(\mathrm{P}=0.040)$, and $\mathrm{BMI}(\mathrm{P}=0.001)$, percentage of lymphocytes $(\mathrm{P}=0.000)$, and NLR $(\mathrm{P}=0.000)$. Female patients accounted for larger proportion in the high level PLR group than the low level PLR group. And in the high PLR group the average level of BMI was lower than that of the low level PLR group. The lower PLR level was correlated with higher percentage of lymphocytes and lower level of NLR.

\section{Selection of Prognostic Factors for CLNM}

According to the results of univariate analysis, age $(p=0.048)$, medicine for diabetes $(p=0.093)$, largest tumor size $(p=0.000)$, duration of diabetes $(p=0.088)$, laterality $(\mathrm{p}=0.001)$, multifocality $(\mathrm{p}=0.000)$, and NLR $(\mathrm{p}=0.053)$ 
Table 2 Correlations Between NLR and Clinical Characteristics in All Cohorts

\begin{tabular}{|c|c|c|c|}
\hline \multirow{2}{*}{ Variables } & NLR $\leq 2.92(\%)$ & NLR >2.92(\%) & \multirow[t]{2}{*}{ P-value } \\
\hline & $N=379(83.1)$ & $N=77(16.9)$ & \\
\hline Age & & & 0.458 \\
\hline$\leq 55(Y)$ & $175(46.2)$ & $32(4 I .6)$ & \\
\hline$>55(Y)$ & $204(53.8)$ & $45(58.4)$ & \\
\hline Sex & & & 0.894 \\
\hline Female & $254(67.0)$ & $5 I(66.2)$ & \\
\hline Male & $125(33.0)$ & $26(33.8)$ & \\
\hline Medicine for diabetes & & & 0.944 \\
\hline Metformin(-) & $210(55.4)$ & $43(55.8)$ & \\
\hline Metformin $(+)$ & $169(44.6)$ & $34(44.2)$ & \\
\hline Laterality & & & 0.867 \\
\hline Unilateral & $312(82.3)$ & $64(83.1)$ & \\
\hline Bilateral & $67(17.7)$ & $13(16.9)$ & \\
\hline Multifocality & & & 0.840 \\
\hline Solitary & $275(72.6)$ & $55(71.4)$ & \\
\hline Multiple & 104(27.4) & $22(28.6)$ & \\
\hline Hashimoto's thyroiditis & & & 0.878 \\
\hline Absent & $337(88.9)$ & $68(88.3)$ & \\
\hline Present & $42(11.1)$ & $9(11.7)$ & \\
\hline $\begin{array}{l}\text { Largest tumor size }(\mathrm{cm}) \text {, median } \\
(\mathrm{IQR})\end{array}$ & $0.7(0.5-1.1)$ & $0.7(0.5-1.2)$ & 0.861 \\
\hline $\begin{array}{l}\text { Duration of } \operatorname{diabetes}(\mathrm{Y}) \text {, median } \\
(\mathrm{IQR})\end{array}$ & $5(2-10)$ & $4(2-10)$ & 0.783 \\
\hline BMI(kg/m2), median (IQR) & $24.77(23.03-26.95)$ & $24.49(22.83-25.97)$ & 0.291 \\
\hline $\mathrm{TC}(\mathrm{mmol} / \mathrm{L})$, median $(\mathrm{IQR})$ & $4.86(4.09-5.6 I)$ & $4.86(3.87-5.72)$ & 0.737 \\
\hline $\mathrm{TG}(\mathrm{mmol} / \mathrm{L})$, median (IQR) & $1.90(1.26-2.87)$ & $1.62(1.18-2.27)$ & 0.100 \\
\hline Glucose(mmol/L), median (IQR) & $7.70(6.30-10.80)$ & $8.30(6.30-11.10)$ & 0.440 \\
\hline Albumin(g/L), median (IQR) & $43.25(40.80-45.30)$ & $43.20(40.00-46.20)$ & 0.736 \\
\hline AGR, median (IQR) & $1.42(1.30-1.60)$ & $1.40(1.30-1.60)$ & $0.56 \mathrm{I}$ \\
\hline $\begin{array}{l}\text { Percentage of lymphocytes, median } \\
\text { (IQR) }\end{array}$ & $0.336(0.292-0.376)$ & $0.195(0.168-0.221)$ & $0.000 *$ \\
\hline $\mathrm{TSH}(\mathrm{mlU} / \mathrm{L})$, median (IQR) & $1.36(0.94-1.92)$ & $1.47(0.97-2.01)$ & 0.438 \\
\hline PLR & & & $0.000 *$ \\
\hline Low level $(\leq 154.70)$ & $334(88.1)$ & $34(44.2)$ & \\
\hline High level (>154.70) & $45(11.9)$ & $43(55.8)$ & \\
\hline CLNM & & & 0.069 \\
\hline CLNM(-) & $220(58.0)$ & $36(46.8)$ & \\
\hline CLNM(+) & $159(42.0)$ & $4 I(53.2)$ & \\
\hline
\end{tabular}

Note: *Means the difference of this variable between two groups was statistically significant. 
Table 3 Correlations Between PLR and Clinical Characteristics in All Cohorts

\begin{tabular}{|c|c|c|c|}
\hline \multirow[t]{2}{*}{ Variables } & PLR $\leq 154.70(\%)$ & PLR > 154.70(\%) & \multirow[t]{2}{*}{ P-value } \\
\hline & $N=368(80.7)$ & $N=88(19.3)$ & \\
\hline Age & & & 0.625 \\
\hline$\leq 55(Y)$ & $165(44.8)$ & $42(47.7)$ & \\
\hline$>55(\mathrm{Y})$ & $203(55.2)$ & $46(52.3)$ & \\
\hline Sex & & & $0.040 *$ \\
\hline Female & $238(64.7)$ & $67(76.1)$ & \\
\hline Male & $130(35.3)$ & $21(23.9)$ & \\
\hline Medicine for diabetes & & & 0.448 \\
\hline Metformin(-) & $20 I(54.6)$ & $52(59.1)$ & \\
\hline Metformin $(+)$ & $167(45.4)$ & $36(40.9)$ & \\
\hline Laterality & & & 0.861 \\
\hline Unilateral & $304(82.6)$ & $72(81.8)$ & \\
\hline Bilateral & $64(17.4)$ & $16(18.2)$ & \\
\hline Multifocality & & & 0.856 \\
\hline Solitary & $267(72.6)$ & $63(71.6)$ & \\
\hline Multiple & $101(27.4)$ & $25(28.4)$ & \\
\hline Chronic lymphocytic thyroiditis & & & 0.953 \\
\hline Absent & $327(88.9)$ & $78(88.6)$ & \\
\hline Present & $4 I(I I .1)$ & $10(11.4)$ & \\
\hline $\begin{array}{l}\text { Largest tumor size }(\mathrm{cm}) \text {, median } \\
(\mathrm{IQR})\end{array}$ & $0.7(0.5-1.1)$ & $0.8(0.5-1.2)$ & 0.441 \\
\hline $\begin{array}{l}\text { Duration of diabetes }(Y) \text {, median } \\
(\mathrm{IQR})\end{array}$ & $4(1.5-9)$ & $5(2-10)$ & 0.235 \\
\hline $\mathrm{BMI}(\mathrm{kg} / \mathrm{m} 2)$, median (IQR) & $24.96(23.12-27.18)$ & $24.06(21.88-25.7 I)$ & $0.001 *$ \\
\hline $\mathrm{TC}(\mathrm{mmol} / \mathrm{L})$, median $(\mathrm{IQR})$ & $4.88(4.05-5.54)$ & $4.82(4.05-5.88)$ & 0.553 \\
\hline $\mathrm{TG}(\mathrm{mmol} / \mathrm{L})$, median $(\mathrm{IQR})$ & $1.92(1.21-2.88)$ & $1.63(1.21-2.30)$ & 0.161 \\
\hline Glucose(mmol/L), median (IQR) & $7.70(6.30-10.85)$ & $8.20(6.40-10.95)$ & 0.376 \\
\hline Albumin $(g / L)$, median (IQR) & $43.40(40.80-45.60)$ & $43.20(40.00-45.40)$ & 0.405 \\
\hline AGR, median (IQR) & $1.40(1.30-1.60)$ & $1.40(1.20-1.60)$ & 0.678 \\
\hline $\begin{array}{l}\text { Percentage of lymphocytes, median } \\
\text { (IQR) }\end{array}$ & $0.333(0.282-0.376)$ & $0.237(0.194-0.304)$ & $0.000 *$ \\
\hline $\mathrm{TSH}(\mathrm{mlU} / \mathrm{L})$, median (IQR) & $1.36(0.96-1.93)$ & $1.41(0.9 \mid-1.74)$ & 0.622 \\
\hline NLR & & & $0.000 *$ \\
\hline Low level $(\leq 2.92)$ & $334(90.8)$ & $45(5 I .1)$ & \\
\hline High level (>2.92) & $34(9.2)$ & $43(48.9)$ & \\
\hline CLNM & & & 0.416 \\
\hline CLNM(-) & $210(57.1)$ & $46(52.3)$ & \\
\hline CLNM(+) & $158(42.9)$ & $42(47.7)$ & \\
\hline
\end{tabular}

Note: *Means the difference of this variable between two groups was statistically significant. 
were statistically related to CLNM. So we integrated these factors into the multivariate logistic regression analysis of which the results indicated that age ( $>55 \mathrm{Y}$ ) [odd-s ratio (OR) $=0.619$, with a $95 \%$ confidence interval $(\mathrm{CI}): 0.394-0.971$, $\mathrm{p}=0.037$ ], multifocality (multiple) [odds ratio $(\mathrm{OR})=2.173$, with a $95 \%$ confidence interval (CI): $1.321-3.575, \mathrm{p}=0.002$ ], largest tumor size $(\mathrm{cm})$ [odds ratio $(\mathrm{OR})=2.427$, with a $95 \%$ confidence interval (CI): 1.642-3.588, $\mathrm{p}=0.000]$, NLR $(>2.92)$ [odds ratio $(\mathrm{OR})=1.856$, with a $95 \%$ confidence interval (CI): $1.023-3.369, \mathrm{p}=0.042]$ were independent prognostic factors of CLNM in PTC patients complicated with T2DM (as shown in Table 4).

\section{Nomogram Construction}

To go a step further, we used the $\mathrm{R}$ software (version 4.0.3) to construct the nomogram for the prediction of CLNM. We included variables that were of statistical significance according to the exclusion criterion of $p$ value $(0.05)$ in the multivariate logistic regression analysis into our nomogram. In order to simplify the predictive model, we consequently turned the largest tumor size from continuous variable to categorical variable by the cut-off value calculated by ROC, of which the best cut-off point was $0.95(\mathrm{~cm})$. The nomogram we constructed is presented in Figure 2. The respective scores of each independent predictive factor were plotted and the total scores can be obtained by adding them up one by one to verify the possibility of CLNM.

\section{Evaluation of Nomogram}

Applying this nomogram we constructed, we calculated each individual's total points and thus obtained the predicted possibility of CLNM. Then we performed the receiver operating characteristic curve to find out the best cutoff value of predicted possibility to discriminate the status of CLNM. And the results of parameters of diagnostic test for applying this nomogram in all cohorts are shown in Table 5. We can tell from the table that we can get a sensitivity of 0.535 , a specificity of 0.797 , a PPV(\%) of 67.7 , and a NPV(\%) of 68.7 when we choose the cutoff value of 0.5273 . We could tell the $\mathrm{C}$-index of the nomogram in the training data set was 0.728 , with a $95 \%$ confidence interval (CI): $0.675-0.782$, as shown in Figure 3A. And great consistencies between predictive values and actual condition were represented in the calibration curves we performed, as shown in Figure 4A (via Hosmer-Lemeshow test, $\mathrm{p}$-value $0.7955961>0.1$ ). To further verify the accuracy of this predictive model, we then collected the clinicopathological data of 100 patients with PTC and T2DM who were from another medical center (Zhejiang Provincial People's Hospital) as the external testing data set. In the external testing data set, the C-index of this nomogram was 0.618 , with a $95 \%$ confidence interval (CI): $0.500-0.735$, as shown in Figure 3B. The calibration curve of external testing data set was shown in Figure 4B (via Hosmer-Lemeshow test, p-value $0.1150245>0.1$ ). According to the p-value calculated via HosmerLemeshow test, of which the p-value exclusion criterion was 0.1 , there were good consistencies between the predicted values and the actually observed one. Decision curve analysis (DCA) for CLNM in PTC patients complicated with $\mathrm{T} 2 \mathrm{DM}$ in training data set and external testing data set was shown in Figure 5A and B respectively. The net benefit was represented by the $y$-axis. The net benefit, by applying this nomogram of predicting CLNM, was represented by the dotted line. And the meaning of the grey line was the net clinical benefit patients got on the assumption that all patients had CLNM and were all treated with central compartment lymph node dissection (CLND). As for the black one, it represented the net clinical benefit patients received on the assumption that none of the patients had CLNM and none of them were treated with central compartment lymph node dissection (CLND). As we can tell from the DCA, applying this nomogram to predict the risk of CLNM occurrence and performing selective CLND according to the predictive possibility calculated by the nomogram and threshold probability before surgery, would be more reasonable and have more clinical net benefit than having all of the patients or none of the patients treated with CLND with a range of the threshold probability between approximately $20 \%$ and $90 \%$ in the training data set, and between approximately $40 \%$ and $90 \%$ in the external testing data set.

\section{Discussion}

As we know, this study is the first one focusing on the association between the preoperative NLR, PLR, and CLNM in PTC patients complicated with T2DM, as numerous studies have revealed that a high level of NLR or PLR was a risk factor for CLNM in patients with PTC. ${ }^{18-20}$ However, the levels of NLR and PLR are different in normal people and people with diabetes mellitus, as we have mentioned previously., ${ }^{5,6}$ Accordingly, there has been no study evaluating the association of NLR and PLR with CLNM in PTC patients complicated with 
Table 4 Univariate and Multivariate Analysis of Logistic Regression in the Training Data Set

\begin{tabular}{|c|c|c|c|c|}
\hline \multirow[t]{2}{*}{ Variables } & \multicolumn{2}{|c|}{ Univariate Analysis of CLNM } & \multicolumn{2}{|c|}{ Multivariate Analysis of CLNM } \\
\hline & OR(95\% Cl) & P-value & OR(95\% Cl) & P-value \\
\hline Age & & 0.048 & & 0.037 \\
\hline$\leq 55(Y)$ & 1 & & 1 & \\
\hline$>55(Y)$ & $0.653(0.428-0.996)$ & & $0.619(0.394-0.971)$ & \\
\hline Sex & & 0.240 & & \\
\hline Female & 1 & & & \\
\hline Male & $\mathrm{I} .304(0.837-2.03 \mathrm{I})$ & & & \\
\hline Medicine for diabetes & & 0.093 & & \\
\hline Metformin(-) & I & & & \\
\hline Metformin(+) & $0.696(0.456-1.063)$ & & & \\
\hline Laterality & & 0.001 & & \\
\hline Unilateral & $\mathrm{I}$ & & & \\
\hline Bilateral & $2.692(I .532-4.731)$ & & & \\
\hline Multifocality & & 0.000 & & 0.002 \\
\hline Solitary & I & & I & \\
\hline Multiple & $2.474(1.539-3.975)$ & & $2.173(1.32 I-3.575)$ & \\
\hline Hashimoto's thyroiditis & & 0.592 & & \\
\hline Absent & 1 & & & \\
\hline Present & $1.197(0.619-2.315)$ & & & \\
\hline Largest tumor size $(\mathrm{cm})$ & $2.606(1.756-3.866)$ & 0.000 & $2.427(1.642-3.588)$ & 0.000 \\
\hline Duration of diabetes $(\mathrm{Y})$ & $0.962(0.920-1.006)$ & 0.088 & & \\
\hline $\mathrm{BMI}(\mathrm{kg} / \mathrm{m} 2)$ & $0.989(0.924-1.058)$ & 0.753 & & \\
\hline $\mathrm{TC}(\mathrm{mmol} / \mathrm{L})$ & $0.895(0.76 \mathrm{I}-1.052)$ & 0.179 & & \\
\hline $\mathrm{TG}(\mathrm{mmol} / \mathrm{L})$ & $1.001(0.899-1.114)$ & 0.989 & & \\
\hline Glucose(mmol/L) & $0.999(0.94|-| .059)$ & 0.962 & & \\
\hline Albumin $(g / L)$ & $0.988(0.942-1.036)$ & 0.617 & & \\
\hline AGR, median (range) & $0.766(0.309-1.899)$ & 0.565 & & \\
\hline Percentage of lymphocytes & $1.095(0.165-7.250)$ & 0.925 & & \\
\hline $\mathrm{TSH}(\mathrm{mlU} / \mathrm{L})$ & $1.025(0.934-1.125)$ & 0.602 & & \\
\hline NLR & & 0.053 & & \\
\hline Low level $(\leq 2.92)$ & I & & I & 0.042 \\
\hline High level $(>2.92)$ & $1.75 \mid(0.994-3.086)$ & & $1.856(1.023-3.369)$ & \\
\hline PLR & & 0.168 & & \\
\hline Low level $(\leq 154.70)$ & 1 & & & \\
\hline High level $(>154.70)$ & $1.437(0.858-2.408)$ & & & \\
\hline NLR+PLR (NPS) & & 0.056 & & \\
\hline Score 0 & I & & & \\
\hline Score I & $1.893(1.086-3.300)$ & 0.024 & & \\
\hline Score 2 & $1.515(0.740-3.102)$ & 0.256 & & \\
\hline
\end{tabular}




\section{Points}

$\begin{array}{lllllllllll}0 & 10 & 20 & 30 & 40 & 50 & 60 & 70 & 80 & 90 & 100\end{array}$

NLR

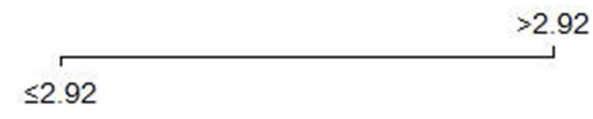

Age

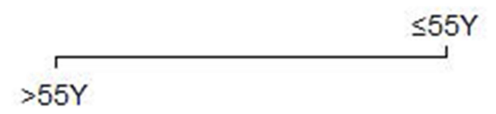

Size

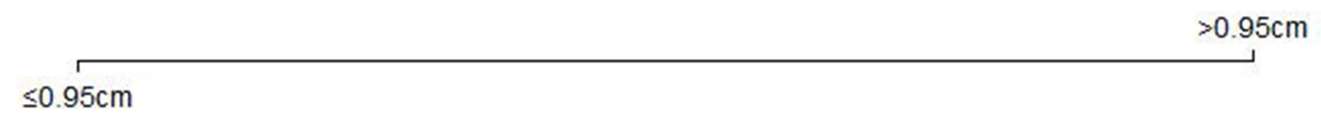

Multifocality

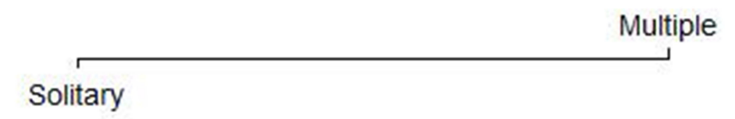

Total Points

$\begin{array}{llllllllllllll}0 & 20 & 40 & 60 & 80 & 100 & 120 & 140 & 160 & 180 & 200 & 220 & 240 & 260\end{array}$

Diagnostic possibility

\begin{tabular}{llllllll}
0.2 & \multicolumn{1}{|c}{} & & & & \\
\hline & 0.3 & 0.4 & 0.5 & 0.6 & 0.7 & 0.8 & 0.9
\end{tabular}

Figure 2 Nomogram predicting the status of central compartment lymph node metastasis in PTC patients with T2DM.

T2DM. Hence, in order to "fill the gaps" in this field, our study still remains worthwhile to be investigated.

Type 2 diabetes mellitus is nowadays known as a chronic disease with high incidence and mortality. ${ }^{1}$ More and more reports have revealed that the levels of inflammatory cytokines in patients with diabetes, such as tumor necrosis factor (TNF)-a, c-reactive protein (CRP), interleukin (IL)-1, etc., are much higher than that of non-diabetic population. ${ }^{2,3}$ And it is widely admitted that chronic inflammation plays an important role in the occurrence and development of diabetes mellitus as well as the pathogenesis of its complications. Of note, more and more evidence has shown that systemic inflammation has significant roles in tumor occurrence, development, progression, metastasis and recurrence in different kinds of solid tumors. Malignant cells can secrete cytokines and chemokines into the peripheral blood circulation and thus trigger the systemic inflammatory response. ${ }^{21}$ Hence, alterations in the organism occur including lymphocytopenia, thrombocytosis, and neutrophilia. A large number of studies have reported that platelets can stimulate angiogenesis and as a result promote the development and progression of primary tumor cells. Meanwhile, it has a strong connection with tumor cell metastasis via invasion of the human organism's immune regulation system. ${ }^{22-24}$ What is more, platelets have been discovered to hinder the natural killer cells to fulfill its ability to inhibit the primary tumor cells. ${ }^{25}$ Furthermore, studies have also found that neutrophils have a significant role in angiogenesis, tumor cell growth, 
Table 5 Parameters of Diagnostic Test for Applying Nomogram in All Cohorts

\begin{tabular}{|l|c|c|c|c|c|c|c|}
\hline Cutoff Value & Sensitivity & Specificity & Youden Index & PPV(\%) & NPV(\%) & FPR(\%) & FNR(\%) \\
\hline 0.3177 & 0.72 & 0.531 & 0.251 & 40.0 & 70.8 & 46.9 & 28.0 \\
\hline 0.3463 & 0.69 & 0.594 & 0.284 & 57.0 & 71.0 & 40.6 & 31.0 \\
\hline 0.4052 & 0.625 & 0.672 & 0.297 & 59.8 & 69.6 & 32.8 & 37.5 \\
\hline 0.4651 & 0.59 & 0.703 & 0.293 & 60.8 & 68.7 & 29.7 & 41.0 \\
\hline 0.4965 & 0.545 & 0.766 & 0.311 & 64.5 & 70.8 & 23.4 & 45.5 \\
\hline 0.5273 & 0.535 & 0.797 & 0.332 & 67.7 & 68.7 & 20.3 & 46.5 \\
\hline 0.5875 & 0.435 & 0.898 & 0.333 & 77.0 & 67.1 & 10.2 & 56.5 \\
\hline 0.6469 & 0.425 & 0.902 & 0.327 & 77.3 & 66.8 & 9.8 & 57.5 \\
\hline
\end{tabular}

Abbreviations: NPV, negative predictive value; PPV, positive predictive value; FPR, false positive rate; FNR, false negative rate.

tumor development, and metastasis, which is similar to platelets. ${ }^{26-28}$ It has also been detected that lymphocytopenia has become an adverse biomarker for overall survival in different human cancers. The role of lymphocytes in the activation of organism cell-mediated immune response and tumor cell lysis is nowadays confirmed to be of great significance. $^{29,30}$

The prognostic values of NLR and PLR have gradually been discovered in various human cancers according to the effects of platelets, neutrophils, and lymphocytes, as mentioned previously. There are some existing studies which have focused on the relation of NLR and PLR with thyroid malignancies. For example, Kocer et $\mathrm{al}^{31}$ discovered that the level of NLR in patients with benign thyroid diseases including lymphocytic thyroiditis and multinodular goiter was significantly lower than that in patients with PTC. And the most objective cutoff-value verified to better distinguish between malignancy and benign thyroid disease was 1.91. Meanwhile, it has been demonstrated that a larger tumor size as well as a higher
A

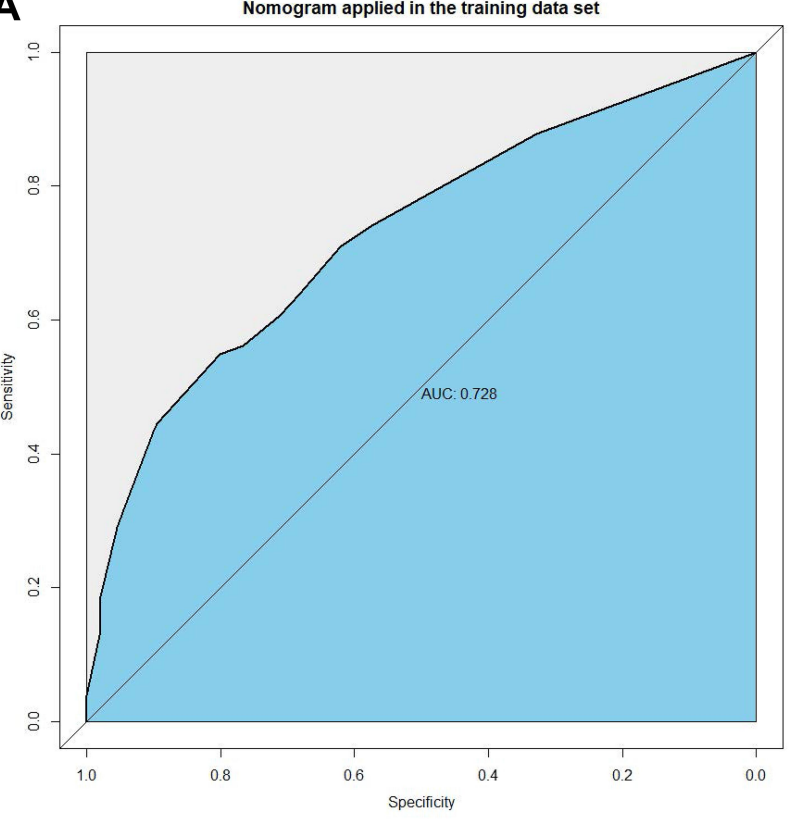

B

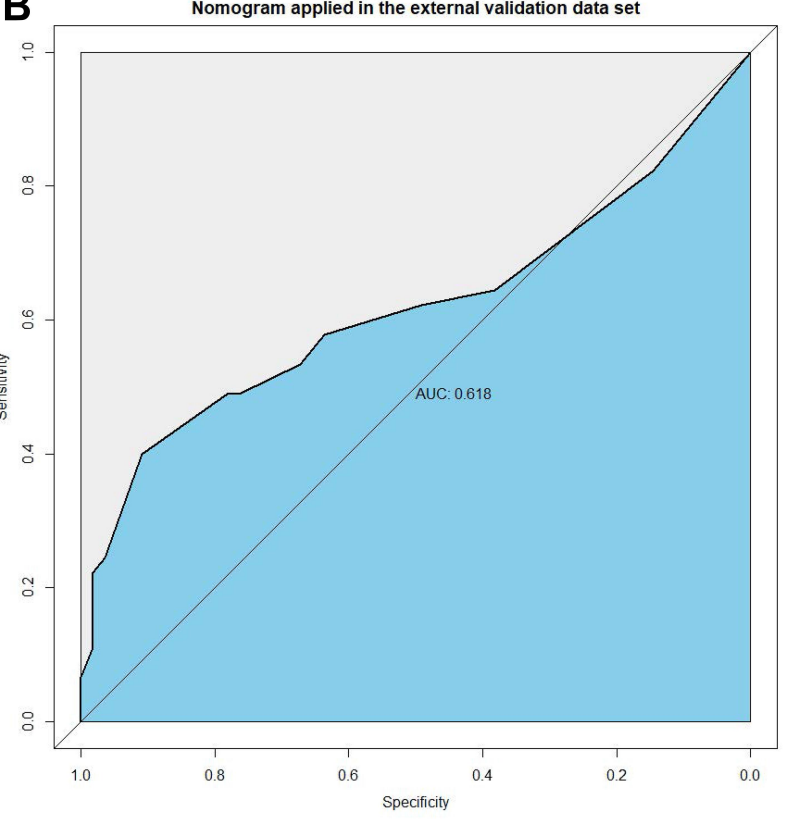

Figure 3 Receiver operating characteristic (ROC) analysis based on the nomogram in the training data set (A), in the external validation data set (B). The AUC of applying this nomogram in the training data set was 0.728 , with a $95 \%$ confidence interval $(\mathrm{Cl}): 0.675-0.782$. The AUC of applying this nomogram in the external validation data set was 0.618 , with a $95 \%$ confidence interval $(\mathrm{Cl}): 0.500-0.735$. 
A

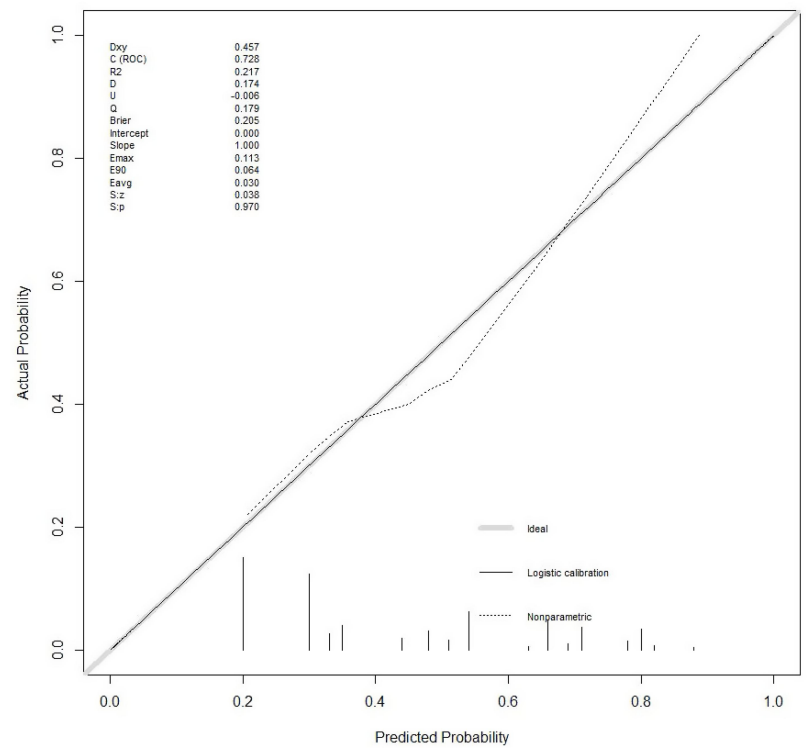

B

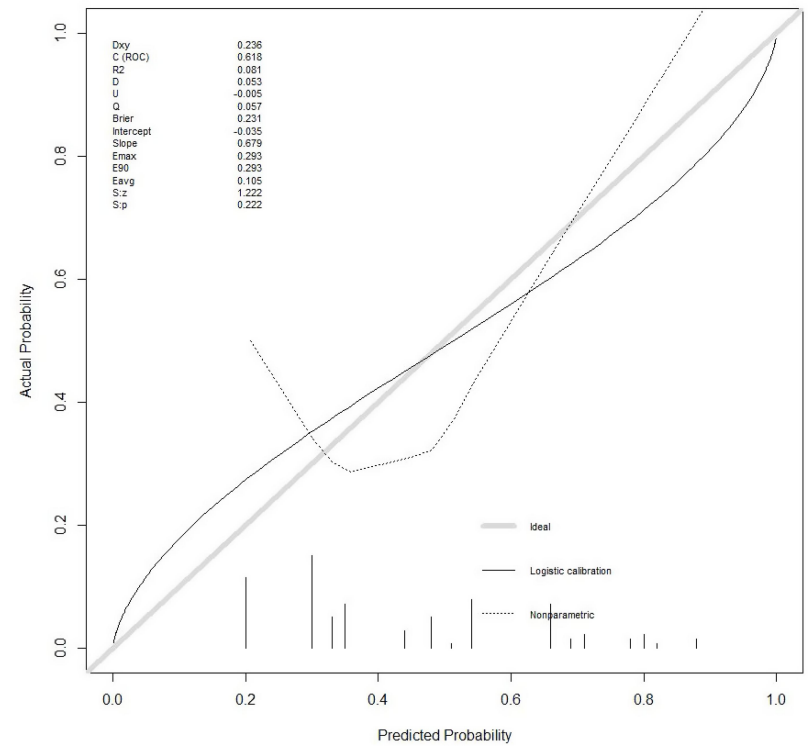

Figure 4 Calibration curve of the nomogram in the training data set (A), in the external validation data set (B). Great consistencies between predictive values and actual condition were represented, as Hosmer-Lemeshow test showed the $\mathrm{p}$-value was 0.7955961 ( $>0.1), 0.1150245(>0.1)$, respectively.

A

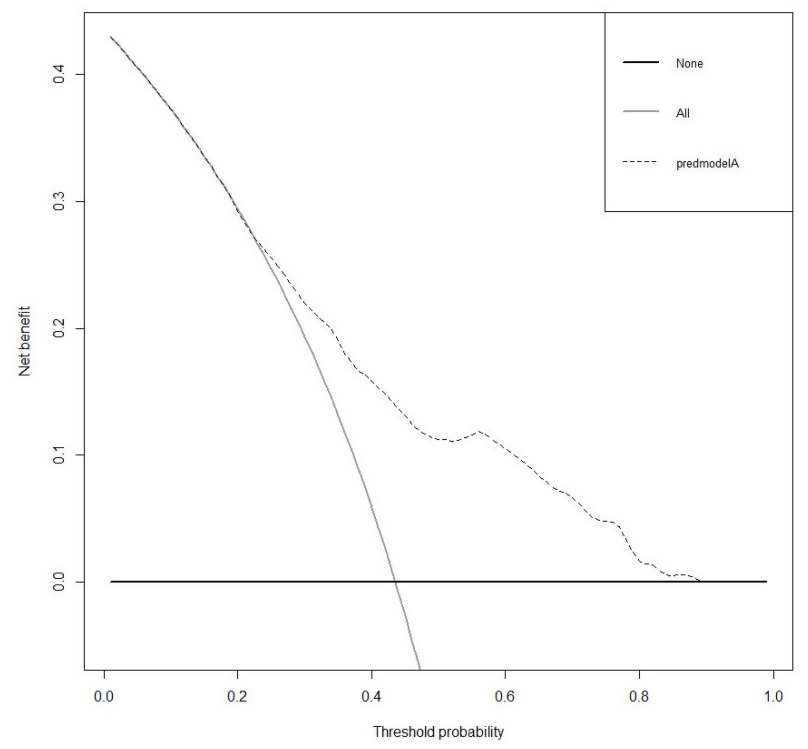

B

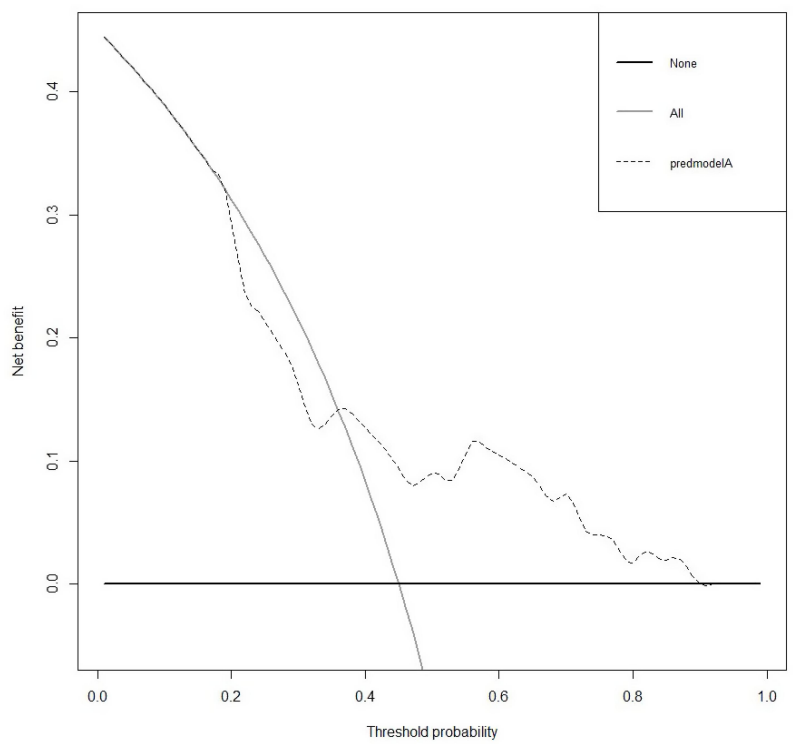

Figure 5 Decision curve analysis (DCA) for applying this nomogram in training data set (A), in external testing data set (B). DCA showed that applying this nomogram will result in more clinical net benefit than having all of the patients or none of the patients treated with CLND with a range of the threshold probability between approximately $20 \%$ and $90 \%$ in the training data set, and between approximately $40 \%$ and $90 \%$ in the external testing data set.

risk of tumor progression and recurrence are strongly connected with a higher NLR, by Liu et al. ${ }^{32}$ In the present study, we found a relation between NLR and PLR $(\mathrm{P}=0.000)$ as well as percentage of lymphocytes $(\mathrm{P}=0.000)$, as the high NLR group (NLR $>2.92)$ had a higher level of PLR and a lower percentage of lymphocytes, which were all statistically significant. However, Kim et $\mathrm{al}^{33}$ found that there was not enough evidence to prove the actual relation between clinicopathological data and NLR in 1066 female PTC patients. On the contrary, they have discovered a statistically significant association between high level of pre-operative PLR and 
the status of lymph node metastasis. In our study, the level of PLR was significantly correlated with $\operatorname{sex}(\mathrm{P}=0.040)$, BMI $(\mathrm{P}=0.001)$, percentage of lymphocytes $(\mathrm{P}=0.000)$, and NLR $(\mathrm{P}=0.000)$. We observed that the female patients accounted for a bigger proportion in the high PLR group (PLR $>154.70)$, and the BMI level of the high PLR group was lower than that of the low PLR group.

Furthermore, as for the standard and validated criteria for cut-off values of NLR, there has been no conclusion in the recent and former literature. The cut-off value of NLR used in the study of Gong et $\mathrm{al}^{34}$ was 2.0, which was different from that of our study. A significant association was found between high NLR and the status of lymph node metastasis, tumor size, and multifocality. Of note, investigators found, in recent studies, that the NLR and PLR values were higher in the diabetic group than in the non-diabetic group, and the reason might be the high and increased insulin resistance. ${ }^{5,6}$ Hence, it might be the reason why the cut-off point verified for NLR in the present study (NLR:2.92) was much higher than that in the other reported study. In addition, what should also arouse attention is that in the studies focusing on solid tumors the NLR values they verified were much higher than those in previous studies which focused on thyroid carcinoma. For instance, in the study of Templeton et al, ${ }^{35}$ they verified the optimal cutoff value of NLR as 4 in a meta-analysis focusing on solid tumors.

In the study of Kim et al, ${ }^{36}$ they revealed that the preoperative levels of NLR and PLR in patients aged $<45$ years were much higher than that in patients who were older and in patients without Hashimoto's thyroiditis, which was statistically significant. They also noticed the incidence of lateral lymph node metastasis (LNM) was significantly increased in the higher level of preoperative PLR group. In our study, we detected that age $>55$ [OR:0.619, 95\% CI (0.394-0.971)] was a protective independent factor for CLNM in PTC patients with T2DM. Kwan et $\mathrm{al}^{37}$ found that the average level of NLR was $1.9 \pm 0.9$ in the group aged $<45$, and $1.7 \pm 0.9$ in the group aged $>45$, which was statistically significant $(\mathrm{p}<0.001)$. And in our study we found that age $>55 \mathrm{Y}$ was a protective factor for CLNM [odds ratio $(\mathrm{OR})=0.619$, with a $95 \%$ confidence interval (CI): 0.394-0.971, p=0.037]. However, the American cancer federation identified age $>45$ years as a risk factor for lymph node metastasis and postoperative local recurrence in patients with thyroid cancer. In 2017, AJCC released the TNM Staging System for Thyroid Cancer (8th Edition), which raised the staging age from 45 years old to 55 years old. ${ }^{38}$

It has been indicated that in operable colorectal and pancreatic cancers, a high level of preoperative PLR in patients was usually considered as a marker of poor prognosis, ${ }^{39}$ which is similar to some other kinds of cancers, such as colorectal, gastric, cholangiocarcinoma, pancreatic, ovarian, and lung carcinomas. ${ }^{40}$ However, the roles of NLR as well as PLR in thyroid carcinoma have only been evaluated by a few studies. It is cheap, efficient, safe, and repeatable to measure the value of PLR and also NLR, which is clinically available. The value of NLR and PLR combined has been found, in a previous study on esophageal squamous cell carcinoma, to be much more applicable to predict postoperative survival rather than focusing on either the NLR or PLR alone. ${ }^{41}$ As a result, in order to figure out if there was better prognostic value of combined NLR and PLR, we assessed the association of combination of NLR and PLR, which we called NPS in the present study, and status of CLNM in patients complicated with T2DM and PTC. Finally, it is unfortunate that the results of our study showed there was no statistically significant connection between NPS and CLNM in PTC patients with T2DM via multi-variate logistic regression analysis, as shown in Table 4.

Recurrent laryngeal nerve (RLN) palsy is a serious complication of thyroid surgery that could significantly decrease a patient's quality of life. Unilateral RLN palsy could cause dysphagia, hoarseness of voice, or respiratory complications due to aspiration; bilateral RLN palsy is a rare and severe complication that may necessitate tracheostomy. $^{42}$ The non-selective prophylactic central lymphadenectomy would increase the risk of RLN injury and subsequently affect the quality of life of patients. Furthermore, DCA showed that applying this nomogram will help patients get more clinical net benefit than having all of the patients or none of the patients treated with central compartment lymph node dissection (CLND) and thus reduce the risk of RLN injury as well. And it has been reported that routine central neck dissection for differentiated thyroid cancer (DTC) to prevent future recurrence is still a matter of discussion, due to the increased risk of injury to parathyroid glands, without clear demonstrable benefits in terms of long-term survival. ${ }^{43}$ Applying this nomogram would also help lower the risk of parathyroid glandinjury and consequently minimize the risk of hypocalcemia. 
Of course there are some limitations to our present study due to retrospective study design, including some missing diabetic-related information including $\mathrm{HbAC}(\%)$, proinsulin C-peptide, as they are not routine tests for patients at the department of Surgical Oncology during admission. Furthermore, optimal and standard cut-off points for NLR and PLR were still unclear according to the recent and former study.

In conclusion, neutrophil-to-lymphocyte ratio (NLR) could easily be applied in clinical practice, and high level of preoperative NLR was an independent predictor for CLNM in PTC patients with T2DM. And the verified optimal cutoff value of NLR in this study was 2.9204 . Applying this nomogram we constructed, will help stratify high-risk CLNM patients, as a result enabling them to receive appropriate measures and get more net clinical benefit. What is more, we hope to find more sensitive indicators in the near future, and add new variables into our nomogram to further improve the sensitivity and specificity of our predictive model.

\section{Ethical Approval and Informed Consent}

The clinical ethics committee approval was given by the ethics committees of the Zhejiang Provincial People's Hospital and the First Affiliated Hospital of Wenzhou Medical University. This study was conducted in light of the Declaration of Helsinki. All individual participants signed written informed consent including participating in this study.

\section{Author Contributions}

All authors made substantial contributions to conception and design, acquisition of data, or analysis and interpretation of data; took part in drafting the article or revising it critically for important intellectual content; agreed to submit to the current journal; gave final approval of the version to be published; and agree to be accountable for all aspects of the work.

\section{Disclosure}

All authors report that there are no conflicts of interest in the present work.

\section{References}

1. Rydén L, Grant PJ, Anker SD, et al. ESC guidelines on diabetes, pre-diabetes, and cardiovascular diseases developed in collaboration with the EASD. Eur Heart J. 2013;34(39):3035-3087.
2. De Rooij SR, Nijpels G, Nilsson PM, et al. Low-grade chronic inflammation in the relationship between insulin sensitivity and cardiovascular disease (RISC) population: associations with insulin resistance and cardiometabolic risk profile. Diabetes Care. 2009;32 (7):1295-1301. doi:10.2337/dc08-1795

3. Garcia C, Feve B, Ferré P, et al. Diabetes and inflammation: fundamental aspects and clinical implications. Diabetes Metab. 2010;36 (5):327-338. doi:10.1016/j.diabet.2010.07.001

4. Bruun JM, Lihn AS, Verdich C, et al. Regulation of adiponectin by adipose tissue-derived cytokines: in vivo and in vitro investigations in humans. Am J Physiol Endocrinol Metab. 2003;285(3):E527E533. doi:10.1152/ajpendo.00110.2003

5. Demirtas L, Degirmenci H, Akbas EM, et al. Association of hematological indicies with diabetes, Impaired glucose regulation and microvascular complications of diabetes. Int $J$ Clin Exp Med. 2015;8(7):1420-1427.

6. Lou M, Luo P, Tang R, et al. Relationship between neutrophil-lymphocyte ratio and insulin resistance in newly diagnosed type 2 diabetes mellitus patients. BMC Endocr Disord. 2015;15(1):4-9. doi:10.1186/s12902-015-0002-9

7. Wang DS, Ren C, Qiu MZ, et al. Comparison of the prognostic value of various preoperative inflammation-based factors in patients with stage III gastric cancer. Tumour Biol. 2012;33:749-756. doi:10.1007/ s13277-011-0285-z

8. Roxburgh CS, McMillan DC. Role of systemic inflammatory response in predicting survival in patients with primary operable cancer. Future Oncol. 2010;6:149-163. doi:10.2217/fon.09.136

9. Takahashi Y, Kawamura M, Hato T, et al. Neutrophil-lymphocyte ratio as a prognostic marker for lung adenocarcinoma after complete resection. World J Surg. 2016;40(2):365-372. doi:10.1007/s00268015-3275-2

10. Zhang WW, Liu KJ, Hu GL, et al. Preoperative platelet/lymphocyte ratio is a superior prognostic factor compared to other systemic inflammatory response markers in ovarian cancer patients. Tumour Biol. 2015;36(11):8831-8837. doi:10.1007/s13277-015-3533-9

11. Wang SC, Chou JF, Strong VE, et al. Pretreatment neutrophil to lymphocyte ratio independently predicts disease-specific survival in resectable gastroesophageal junction and gastric adenocarcinoma. Ann Surg. 2016;263(2):292-297. doi:10.1097/SLA.0000000000001189

12. Xiao WK, Chen D, Li SQ, et al. Prognostic significance of neutrophil-lymphocyte ratio in hepatocellular carcinoma: a meta-analysis. BMC Cancer. 2014;14(1):117. doi:10.1186/14712407-14-117

13. Chen ZY, Raghav K, Lieu CH, et al. Cytokine profile and prognostic significance of high neutrophil-lymphocyte ratio in colorectal cancer. Br J Cancer. 2015;112(6):1088-1097. doi:10.1038/bjc.2015.61

14. Duan H, Zhang X, Wang FX, et al. Prognostic role of neutrophil-lymphocyte ratio in operable esophageal squamous cell carcinoma. World J Gastroenterol. 2015;21:5591-5597. doi:10.3748/wjg.v21.i18.5591

15. Templeton AJ, Ace O, McNamara MG, et al. Prognostic role of platelet to lymphocyte ratio in solid tumors: a systematic review and meta-analysis. Cancer Epidemiol Biomarkers Prev. 2014;23 (7):1204-1212.

16. Stotz M, Gerger A, Eisner F, et al. Increased neutrophil-lymphocyte ratio is a poor prognostic factor in patients with primary operable and inoperable pancreatic cancer. Br J Cancer. 2013;109(2):416-421. doi:10.1038/bjc.2013.332

17. Kocer D, Karakukcu C, Karaman H, Gokay F, Bayram F. Gokay $\mathrm{F}$ and Bayram F: may the neutrophil/lymphocyte ratio be a predictor in the differentiation of different thyroid disorders? Asian Pac J Cancer Prev. 2015;16(9):3875-3879.

18. Wang Y, Guan Q, Xiang J. Nomogram for predicting central lymph node metastasis in papillary thyroid microcarcinoma: a retrospective cohort study of 8668 patients. Int J Surg. 2018:1;S1743919118307714. 
19. Ning L, Ju-hua H, Song C, Yang L-C, Zhang H-J, Li Z-H Nomogram Including Elastography for Prediction of Contralateral Central Lymph Node Metastasis in Solitary Papillary Thyroid Carcinoma Preoperatively. Cancer Manag Res. 2020;12:10789-10797. doi:10.2147/CMAR.S278382

20. Ceylan Y, Kumanlolu K, Oral A, et al. The Correlation of Clinicopathological Findings and Neutrophil-to-Lymphocyte and Platelet-to-Lymphocyte Ratios in Papillary Thyroid Carcinoma[J]. Mol Imaging Radionucl Ther. 2019;28(1):15-20. doi:10.4274/mirt. galenos.2018.60490

21. Salazar-Onfray F, Lopez MN, Mendoza-Naranjo A. Paradoxical effects of cytokines in tumor immune surveillance and tumor immune escape. Cytokine Growth Factor Rev. 2007;18(1-2):171-182. doi:10.1016/j.cytogfr.2007.01.015

22. Kim PY. Platelets: connecting clotting and lysis. Blood. 2015;125 (16):2459. doi:10.1182/blood-2015-03-632158

23. Jain S, Harris J, Ware J. Platelets: linking hemostasis and cancer. Arterioscler Thromb Vasc Biol. 2010;30(12):2362-2367. doi:10.1161/ ATVBAHA.110.207514

24. Garraud O, Cognasse F. Are platelets cells? And if yes, are they immune cells? Front Immunol. 2015;6:70. doi:10.3389/ fimmu. 2015.00070

25. Nieswandt B, Hafner M, Echtenacher B, et al. Lysis of tumor cells by natural killer cells in mice is impeded by platelets. Cancer Res 1999;59:1295-1300.

26. Balkwill F, Mantovani A. Inflammation and cancer: back to Virchow?. Lancet. 2001;357:539-545.

27. Tavares-Murta BM, Mendonça MAO, Duarte NL, et al. Systemic leukocyte alterations are associated with invasive uterine cervical cancer. Int J Gynecol Cancer. 2010;20:1154-1159. doi:10.1111/ IGC.0b013e3181ef8deb

28. Pichler M, Hutterer GC, Stoeckigt C, et al. Validation of the pretreatment neutrophil-lymphocyte ratio as a prognostic factor in a large European cohort of renal cell carcinoma patients. $\mathrm{Br} J$ Cancer. 2013;108:901-907. doi:10.1038/bjc.2013.28

29. Mehrazin R, Uzzo RG, Kutikov A, et al. Lymphopenia is an independent predictor of inferior outcome in papillary renal cell carcinoma. Urol Oncol. 2015;33:388e319. doi:10.1016/j. urolonc.2014.06.004

30. Wu ES, Oduyebo T, Cobb LP, et al. Lymphopenia and its association with survival in patients with locally advanced cervical cancer. Gynecol Oncol. 2016;140(1):76-82. doi:10.1016/j.ygyno.2015.11.013

31. Kocer D, Karakukcu C, Karaman H, et al. May the neutrophil/ lymphocyte ratio be a predictor in the differentiation of different thyroid disorders? Asian Pac J Cancer Prev. 2015;16 (9):3875-3879. doi:10.7314/APJCP.2015.16.9.3875

32. Liu J, Du J, Fan J, et al. The neutrophil-to-lymphocyte ratio correlates with age in patients with papillary thyroid carcinoma. ORL. 2015;77(2):109-116. doi:10.1159/000375534
33. Kim SM, Kim EH, Kim BH, et al. Association of the Preoperative Neutrophil-to-lymphocyte Count Ratio and Platelet-to-Lymphocyte Count Ratio with Clinicopathological Characteristics in Patients with Papillary Thyroid Cancer. Endocrinol Metab. 2015;30:494-501. doi:10.3803/EnM.2015.30.4.494

34. Gong W, Yang S, Yang X, Guo F. Blood preoperative neutrophil-tolymphocyte ratio is correlated with TNM stage in patients with papillary thyroid cancer. Clinics. 2016;71:311-314. doi:10.6061/ clinics/2016(06)04

35. Templeton AJ, Ace O, McNamara MG, et al. Prognostic Role of Platelet to Lymphocyte Ratio in Solid Tumors: a Systematic Review and Meta-Analysis. Cancer Epidemiol Biomarkers Prev. 2014;23:1204-1212. doi:10.1158/1055-9965.EPI-14-0146

36. Mi KS, Heui KE, Hyun KB, et al. Association of the Preoperative Neutrophil-to-ymphocyte Count Ratio and Platelet-to-Lymphocyte Count Ratio with Clinicopathological Characteristics in Patients with Papillary Thyroid Cancer[J]. Endocrinol Metab. 2015;30:4.

37. Lee KH, Seok EY, Kim EY, et al. Different prognostic values of individual hematologic parameters in papillary thyroid cancer due to age-related changes in immunity[J]. Ann Surg Treat Res. 2019;96 (2):2. doi:10.4174/astr.2019.96.2.70

38. Amin M, Greene F, Edge S, et al. The Eighth Edition AJCC Cancer Staging Manual: continuing to build a bridge from a populationbased to a more "personalized" approach to cancer staging: the Eighth Edition AJCC Cancer Staging Manual. CA. 2017;67 (2):93-99. doi:10.3322/caac. 21388

39. Smith RA, Bosonnet L, Raraty $M$, et al. Preoperative platelet-lymphocyte ratio is an independent significant prognostic marker in resected pancreatic ductal adenocarcinoma. Am J Surg. 2009;197(4):466-472. doi:10.1016/j.amjsurg.2007.12.057

40. Liu CL, Lee JJ, Liu TP, Chang YC, Hsu YC, Cheng SP. Blood neutrophil-to-lymphocyte ratio correlates with tumor size in patients with differentiated thyroid cancer. J Surg Oncol. 2013;107:493-497. doi:10.1002/jso. 23270

41. Feng JF, Huang Y, Liu JS. Combination of neutrophil lymphocyte ratio and platelet lymphocyte ratio is a useful predictor of postoperative survival in patients with esophageal squamous cell carcinoma. Onco Targets Ther. 2013;6:1605-1612. doi:10.2147/OTT.S52501

42. Calò PG, Medas F, Conzo G, et al. Intraoperative neuromonitoring in thyroid surgery: is the two-staged thyroidectomy justified?[J]. Int J Surg. 2017;41:S13-S20. doi:10.1016/j.ijsu.2017.02.001

43. Docimo G, Tolone S, Ruggiero R, et al. Total thyroidectomy without prophylactic central neck dissection combined with routine oral calcium and vitamin D supplements: is it a good option to achieve a low recurrence rate avoiding hypocalcemia? A retrospective study[J]. Minerva Chirurgica. 2013;68(3):321.

\section{Publish your work in this journal}

Cancer Management and Research is an international, peer-reviewed open access journal focusing on cancer research and the optimal use of preventative and integrated treatment interventions to achieve improved outcomes, enhanced survival and quality of life for the cancer patient.
The manuscript management system is completely online and includes a very quick and fair peer-review system, which is all easy to use. Visit http://www.dovepress.com/testimonials.php to read real quotes from published authors. 